\title{
Automatic Generation of Digital Twin Based on Scanning and Object Recognition
}

\author{
Markus SOMMER ${ }^{\mathrm{a}}$, Josip STJEPANDIĆ ${ }^{\mathrm{b} 1}$, Sebastian STOBRAWA ${ }^{\mathrm{c}}$ and Moritz von \\ SODEN $^{\mathrm{d}}$ \\ ${ }^{a}$ isb - innovative software business GmbH, Germany \\ ${ }^{b}$ PROSTEP AG, Germany \\ ${ }^{c}$ Leibniz University Hannover, Institute of Production Engineering and Machine Tools, \\ Germany \\ ${ }^{d B o r n e m a n n ~ G e w i n d e t e c h n i k ~ G m b H ~ \& ~ C o . ~ K G, ~ G e r m a n y ~}$
}

\begin{abstract}
Digital Twin has been recognized as a strategic approach in the modern manufacturing industry to improve both the flexibility and the efficiency. To efficiently generate the Digital Twin of an existing real object in the factory, powerful methods are necessary. Hereby, a fast data acquisition including object recognition and model reconstruction methodology has been combined to resolve these issues. Such a data set often has to replace the missing original digital model. Subsequently, a model reconstruction plan has to be derived so that an editable CAD model, which fulfils process requirements, can be generated using standard geometry creation tools. Such a reverse-engineered CAD model preferably contains form-feature based design intent and can be easily modified due to new design and manufacturing constraints. The presented paper describes an industrial approach for a commercial service being in the implementation to generate the Digital Twin based on fast scanning on a factory.
\end{abstract}

Keywords. Digital Twin, Digital Factory, Object Recognition, Indoor Object Acquisition, Simulation

\section{Introduction}

The digital factory has already been recognized as a strategic advantage by the industry more than ten years ago. The results of a study conducted by the Fraunhofer IPA show the successful application of tools and methods of the digital factory by over twenty percent of the more than a hundred interviewed German midsized companies and major industries since the year 2005. Barely ninety percent of the interviewed companies stated that they planned to deal with the issue digital factory during the next years. An essential component of the digital factory are digital models of products, processes and resources, which aim the standardization of processes, the increasing of flexibility and shortening of the lead time [1]. Especially for digital models in production, there are already existing many fields today (e. g. planning of factories, optimization of layout of the shop floor, approval processes in reconstruction and fire protection or optimization of production processes). According to Wenzel et al., especially simulation is a core element of the digital factory and it is increasingly gaining importance in digitization [2]. Scientifically,

\footnotetext{
${ }^{1}$ Corresponding Author, Email: josip.stjepandic@opendesc.com.
} 
simulation in the fields of production and logistics has been researched and established for a long time [2][3][4]. According to Nyhuis et al. [5], their application supports factories with the optimization of logistic target figures, e. g., concerning adherence to schedules, lead time, performance, stock and costs. Thus, the benefit in the field of material flow planning is assessed as being high or very high [6]. Assuming the gap between the real and the virtual factory, concrete solutions are needed for the continuous synchronization of the planning data and simulation models from the product development, the production planning and the production by using measured values from the real factory [7]. High quality CAD data of all geometrical objects in all stages of planning process are the pre-requisite for seamless downstream processes [8].

With fast scans of the production and subsequent object recognition, the production layout (e. g. size and location of the objects) and the production logic (e. g. machine types, transport routes) can be recorded as automated as possible and visualized true to scale in digital models [9][10][11]. The identification of CAD models from a reference library and the transfer of geometry and other object data (e. g. machine types) as modular objects directly from the library significantly reduce the scan times for a first rough "prescan" of the production [12][13]. At the same time, database reconciliation enables the use of simpler and cheaper scanning methods [14][15]. The definition of suitable interfaces enables the transfer of information into a program for simulation of production systems and a precisely fitting Digital Twin of the manufacturing can be generated - almost without manual interventions [16][17].

The remainder of this paper is structured as follows: in Section 1 the background is given, followed by the description of the general concept in Section 2. In Section 3 the use case is demonstrated with the related discussion in Section 4. Finally, Section 5 summarizes the conclusions and outlook.

\section{Background}

Digital Twin has few pre-requisites. The latest studies prove that using simulation models in manufacturing [18] (hereafter also named „Digital Twin in manufacturing”) is not yet standard in small and medium-sized companies (SME). Causal for this are mainly the following obstacles [18][19][20]:

1. Ambiguous purchasing costs (e. g., owing to manual or inefficient creation of the Digital Twin).

2. Missing knowlegde in the IT-field (e. g., owing to inefficient or too expensive services).

3. Non predictable operating costs (e. g., owing to manual or inefficient adaptation of the Digital Twin).

4. Missing information concerning available simulation tools and application areas as well as the achievable advantage.

Next to the Digital Twin addressed in this article, in literature as well exists the term of the Digital Shadow. The Digital Twin is characterized by the most accurate physical display of the creation in a model. In contrast, according to Schuh et al. [21], the Digital Shadow just presents ,a sufficiently exact image of the relevant data in production, development and associated areas". Thus, the Digital Shadow merely requires process relevant data. To correct the obstacles mentioned above, approaches for automated generation of digital manufacturing twins in the form of simulation models have been 
discussed in literature for more than twenty years. Thereby, numerous references based on the combination of existing technology components can be found. However, other references pursue data driven model generation [22]. Significant research papers that can be found in literature are recapped in the following Table 1:

Table 1. Review of previous research.

\begin{tabular}{|c|c|}
\hline Source & Summary of Key Developments \\
\hline [23] & $\begin{array}{l}\text { - Creation of simulation layouts based on CAD Data } \\
\text { - Direct user requests or integration of special data pools to involve information that } \\
\text { are not derivable from the layout }\end{array}$ \\
\hline [24] & $\begin{array}{l}\text { - Integration of CAD- and system load data (e. g. from product planning and control } \\
\text { systems, shop floor control systems or machine data) } \\
\text { - } \quad \text { Transfer into STEP-based data format and creation of a simulation model }\end{array}$ \\
\hline$[25]$ & $\begin{array}{l}\text { - Model generation based on a proprietary information model } \\
\text { - No use of IT data pools } \\
\text { - Maintenance of the information model's data base via assistance programs using } \\
\text { input validation }\end{array}$ \\
\hline$[26]$ & $\begin{array}{l}\text { - Filling of a simulation data base via filtering and collection, export and manual } \\
\text { addition of manufacturing data } \\
\text { - Model creation by means of defined strategies for an automated interpretation of } \\
\text { manufacturing data } \\
\text { - Development of a prototype is not suitable for productive application }\end{array}$ \\
\hline [27] & $\begin{array}{l}\text { - Supplementation of existing approaches for the automated model generation with } \\
\text { generic variants and logistics components adapted to variant production } \\
\text { - Use of the XML format as neutral data exchange format } \\
\text { - No integration of layout information } \\
\text { - Arrangement of the elements based on a heuristic for non-overlapping structure } \\
\text { within simulation components }\end{array}$ \\
\hline$[19]$ & $\begin{array}{l}\text { - Development of a methodology for partially automated generation of simulation } \\
\text { models } \\
\text { - Collection of the necessary data from different operational information systems } \\
\text { via the design of a data framework to illustrate basic simulation entities in XML } \\
\text { and development of interface modules for selected data sources }\end{array}$ \\
\hline$[22]$ & $\begin{array}{l}\text { - Production of a comprehensive framework for the automation of simulation } \\
\text { - Integration of different data sources via the Core Manufacturing Simulation Data } \\
\text { (CSMD) Information Model }\end{array}$ \\
\hline
\end{tabular}

The process of digitizing a part and creating a CAD model from a scanned point cloud data is feasible if it consumes less time and provides higher accuracy than the manual process by measuring the singular objects in the factory and designing those objects from scratch in CAD. The provision of an adaequate data quality is an important constraint [8]. The typical procedure from process industry is depicted in Figure 1.

In the first step (left), the data acquisition of a object is conducted at customer site, which could be everywhere, by fixed or portable scanner and data are sent to a data management center which provides the data clean-up, the translation of point cloud to $\mathrm{CAD}$ objects (surface, solid and feature) and object upgrade by feature recognition. Finally, the design and project departments need such data set to make new design or design change in a retrofit of the existing object. 


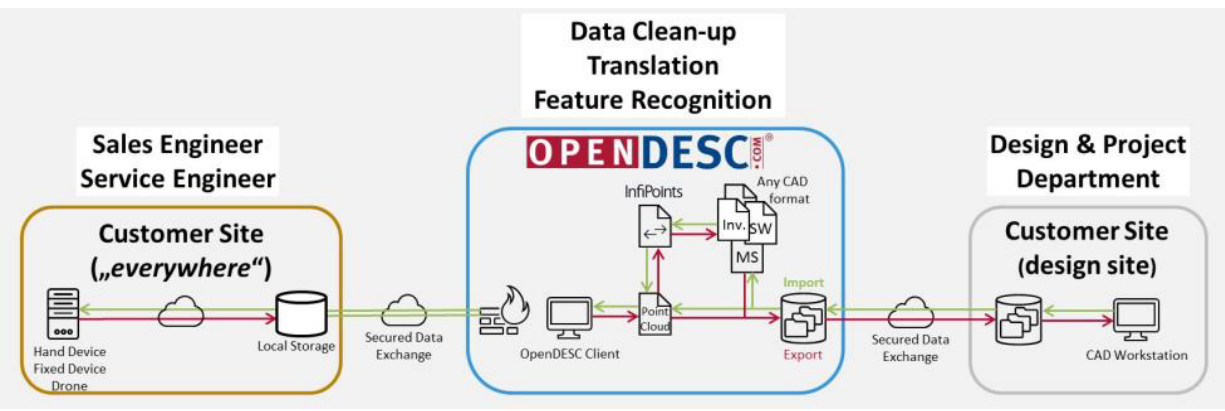

Figure 1. Process chain reverse engineering in the process industry.

\section{Use cases}

Prior to each process and method development, an detailed analysis of the relevant use cases is necessary. Here, various planning activies in a built environment lie in the foreground (Table 2). Each factory and each building have their own history and life cycle. Most commonly, the status as-built is poorly documented and must be thorougly captured in case of change, reorganization and retrofit [10].

Six basic use cases have been identified at all (Table 2). All cases include a capture of the entire factory hall. Due to the high complexity of the subsequent steps, an exact 3D model enriched with the object specific information is a pre-requisite for planning activities in all cases. Process automation is desired both for saving of costs and speedup of the process.

Table 2. Considered use cases.

\begin{tabular}{ll}
\hline Use case & Short Description \\
\hline Factory planning & $\begin{array}{l}\text { Reorganization of structures based on new concepts (e. g. push vs. pull), } \\
\text { layout planning or material flow analyzes. }\end{array}$ \\
\hline Investment planning & $\begin{array}{l}\text { Integration of robotics, purchase of new machines, integration of new } \\
\text { technologies (e. g. 3D print). }\end{array}$ \\
\hline Bottleneck analysis & Identification of workplaces, machines or stations with critical load. \\
\hline Capacity planning & $\begin{array}{l}\text { Comparison of capacity requirements with actually available capacity to } \\
\text { optimize capacity planning. }\end{array}$ \\
\hline Inventory management & Planning and optimizing the stocking of products and materials. \\
\hline In-house material transport & Planning and optimization of the material supply. \\
\hline
\end{tabular}

Provision of adequate 3D models and additional specific information of the factory equipment by their manufacturer is a standard at this time. Usually, models are delivered in neutral and native formats. For the intended purpose, cooperation with the manufacturers of the machine tools has been established to get appropriate and accurate 3D models. For older objects which does not have 3D documentation, an alternative approach need to be developed to derive a feature-based model, e. g. by recognition of singular features. 


\section{Solution concept}

To solve the presented obstacles and unlike the works displayed in literature, this paper introduces a method allowing a preferably automated generation of a simulation model. The development of the method occurs in the project „DigiTwin“. Thereby, the practical relevance of the project becomes apparent through the consortium with the companies isb - innovative software business $\mathrm{GmbH}$, PROSTEP AG and Bornemann Gewindetechnik GmbH \& Co. KG, accompanied by Institute for Production Technology and Machine Tools (IFW) of the Leibniz University of Hannover. The method rests upon the use of expeditious scans of the production and downstream object detection. Thus, the production layout (e. g., size and location of the objects) and the production logistics such as machine type and itinerary, at best are automatically recorded and displayed in models true to scale. Figure 2 shows this context. Thus, three different input parameter groups for the creation of the Digital Twin are introduced.

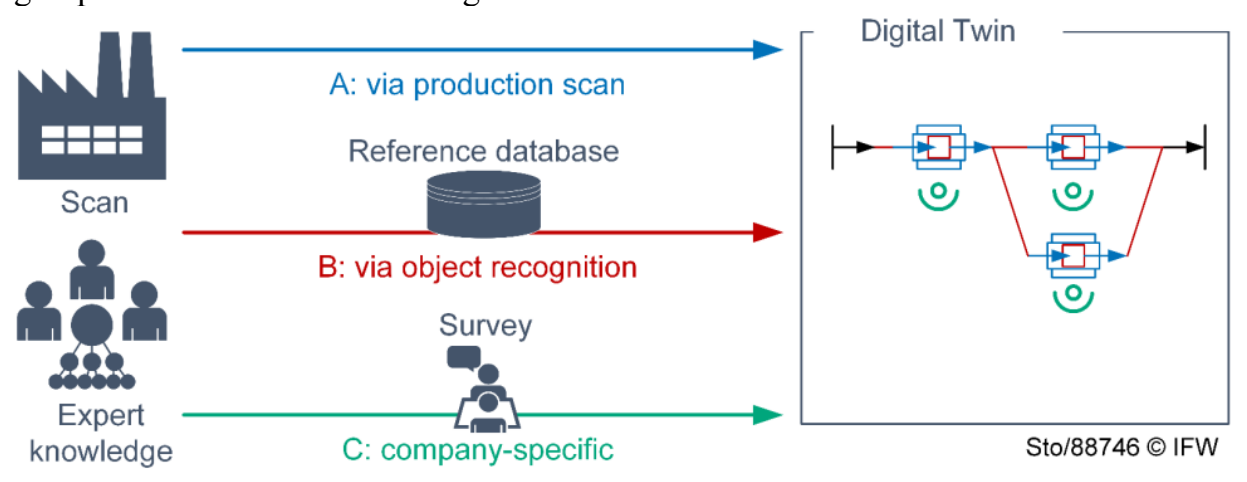

Figure 2. Mapping of relevant information for the Digital Twin.

Parameters of group A (pictured blue in Figure 2) can be determined directly from the scan (e. g., machine geometries). The parameters of group B require additional object detection (e. g., machine types, marked red in Figure 2). To register these parameters, next to the construction scan, a reference data base is required. A comparison of the scanned object with the CAD models of the reference data base accordingly delivers the required input parameters for the Digital Twin. Thus, the parameters of the groups A and B can be recorded automatically. Finally, the parameters of group C (green in Figure 2) are localized company-specific, as they are individual expressions of the company that cannot be recorded generically or automated (e. g., machine ID). To record these parameter groups, according forms are created, so that typical parameters can be surveyed and, at least, this process can be executed with low effort.

The general procedure for an automatic preparation of a Digital Twin as the solution process from a built environment in the factory to the Digital Twin is roughly depicted in the Figure 3. It consists of three fundamental steps: scan, modeling and simulation modeling. Modeling need to be heavily supported by object recognition to save time which would be spent in the step of manual remastering. The object parameter (e. g. machine characteristics) are stored in the CAD library and an external database. Scalability is an important requirement for this approach because theoretically each of the infinite built objects need to be recognized. The expert knowledge of the built environment need to be acquired by forms or expert interviews and also inserted in the simulation process. 


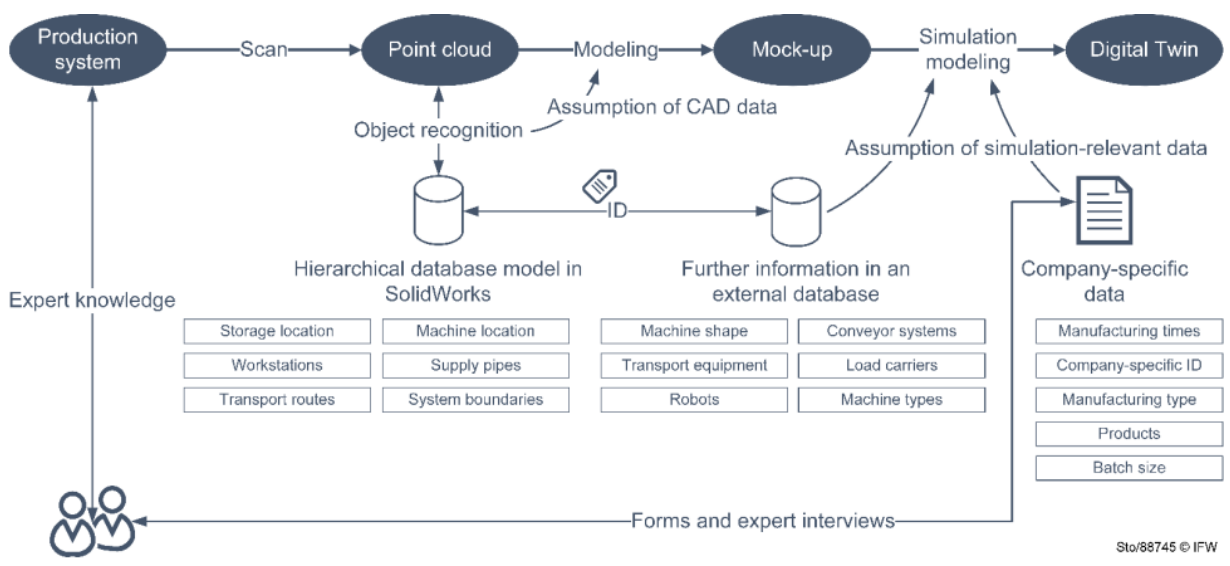

Figure 3. Solution process for the build-up of the Digital Twin.

At this time, the most time-consuming step in this process is the 3D modeling of the factory layout, which need to be substantially simplified and optimized. It is partially determined by the scan procedure which should provide a dense point cloud of high accuracy. The aims of a scan is to generate point cloud models or generate point clouds with high quality for subsequent steps [28].

To fullfil the application requirements by point cloud, these steps are needed:

- point cloud preprocessing,

- mesh reconstruction,

- point cloud segmentation and

- point cloud modeling.

Generally, point cloud preprocessing contains four sub-steps, i. e. registration, noise filtering, outlier removal and down-sampling. It deserves to explain that which of the four substeps are needed depends on the application requirements and the quality of the point clouds. For example, if the application requirement is a combined point cloud and the existing point clouds are separate, then registration is required [29].

A point cloud model of a hole factory is a huge model of dozen of Gigabyte memory space which is challenging and time-consuming from the basic data handling on. Therefore, it looks beneficial to split it into multiple sections according to the structure and layout of the factory. Afterwards, the singular pieces can be considerer as component of an assembly. It makes the work easier and simplifies the distributed work and use of the parallel algorithms. Despite of desire for high process automation, this work will be done manually by using the standard CAD software.

For recognition of a dedicated object in a section of a factory, deep learning (DL) based method are used. DL techniques have emerged as powerful methods for learning features automatically from data [30]. In particular, these techniques have provided significant improvement for object detection, a problem which has attracted enormous attention in the last few years, even though it has been studied for decades before [31].

Object detection and recognition has a wide range of applications in many areas of artificial intelligence and information technologies, including robot vision, consumer electronics, security, autonomous driving, human computer interaction, content based image retrieval, intelligent video surveillance and augmented reality [30]. However, there are certain difficulties in using DL to process $3 \mathrm{D}$ point clouds [28] which must be taken into account: 
- The point cloud is not in a regular format. Unlike an image, a point cloud is a set of points distributed in space. Hence, it is non-grid data. However, typical convolutional architectures can merely deal with highly regular data formats, and it is, therefore, hard to use convolutional neural networks to process raw point clouds.

- The point cloud is unordered. There are various orderings of points, indicating that there are many matrix representations of a particular point cloud. Therefore, how to adapt the changes of the waypoints are arranged is a problem in point cloud processing.

- Three-dimensional point clouds normally contain only the spatial coordinates of points, lacking rich textures, colors, and other information.

Basically, two different methods are used for objects recognition in point cloud data gathered with a scanner: point-based and voxel-based. PointNet, as a point based method, is a DL architecture that is used in 3D object classification and segmentation. It takes point clouds directly as inputs and outputs [31]. In contrast, a 3D detection framework (VoxNet, VoxelNet, LightNet) transforms the point cloud into voxels that each contain a small amount of points. It produces bounding boxes based on the features of the voxels. Finally, a combined approach, called FusionNet, uses both volumetric and pixel information to recognize $3 \mathrm{D}$ objects. These two approaches require different labelling and point cloud pre-processing.

The PointNet and VoxNet architectures demonstrate two ways of detecting objects in a scene. PointNet attempts to semantically segment each point in the data by learning the local and global features of the points and classifying each of them. Clusters of points with the same labels can be detected as objects. VoxNet similarly detects the features of the points using voxels, 3D boxes of points, and learns their properties [32]. Combining a convolutional network and region proposal network, VoxNet can predict 3D bounding boxes of the detected objects.

For this approach, VoxNet has been selected as a basis approach for object recognition for several reasons, altough it does not provide the highest accuracy:

(a) the stable, open-access framework proven by a large community at GitHub,

(b) availability of several implementations by different authors, and

(c) the lowest number of parameters for training which allows low computing time to be expected.

At this time, the real-time processing and recognition is a desirable option, but not a mandatory one. The use of a further architecture will be considered based on the first results with VoxNet.

When it comes to using DL routines, it is necessary to first collect a large amount of data in order to execute the learning phase. This phase can constitute a major bottleneck to an practical engineering application and may have prohibitive costs. Especially in the context of applications with which the amount of input-data needs to be quite large. To provide statistical robustness for this approach, open-source databases such as ShapeNet, ModelNet and DMUNet were used to augmenting thetest base [30].

VoxNet operates on probabilistic occupancy grids, in which each voxel contains the probability that a certainvoxel is occupied in this space. Occupancy grids represent the state of the environment as a 3D lattice of random variables (each corresponding to a voxel) and maintain a probabilistic estimate of their occupancy as a function of incoming sensor data and prior knowledge. A benefit of this approach is that it allows the network to differentiate between voxels that are known to be free and voxels whose occupancy is unknown (e. g., voxels behind where a scan ray hits). 
VoxNet's architecture itself is fairly straightforward, consisting of two convolutional layers, a max pooling layer, and two fully-connected layers to compute an output class score vector (Figure 4) [32]. This network has been selected by stochastic search over hundreds of possible convolutional neural network $(\mathrm{CNN})$ architectures. It is much shallower and has far fewer parameters than most state-of-the-art image classification networks. Conv $(f, d, s)$ indicates $f$ filters of size $d$ and at stride $s, \operatorname{Pool}(m)$ indicates pooling with area $m$, and Full( $n)$ indicates fully connected layer with $n$ outputs. The changes aimed to reduce the number of parameters and increase computational efficiency, making the network easier and faster to learn. The model has 921,736 parameters, most of them from inputs to the first dense layer.

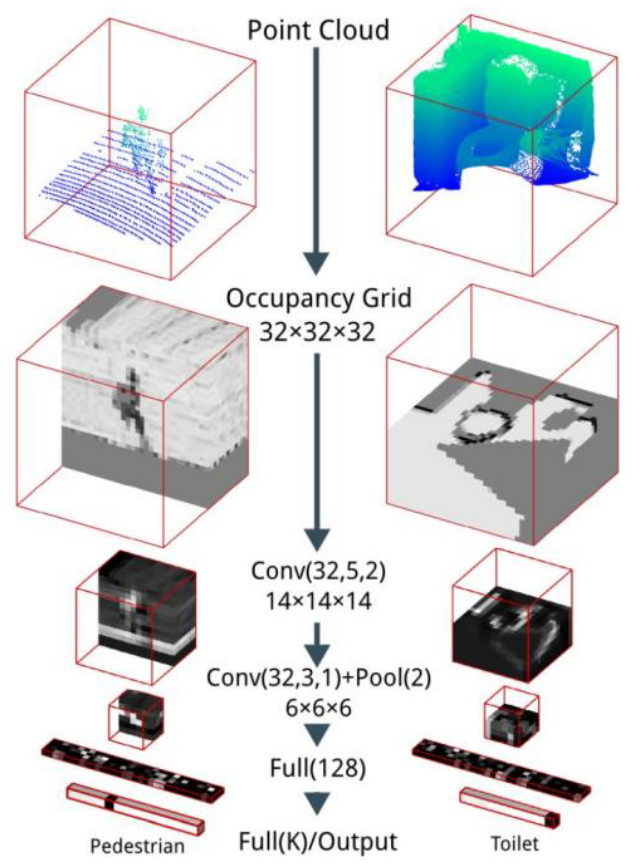

Figure 4. The VoxNet architecture [32].

After an object has been recognized, a corresponding CAD model from the library is inserted as a component in an assembly which represents the factory. This procedure is repeated as long as necessary. The remaining object should be processed using the CAD functions of SolidWorks preferrably embedded in macros.

\section{Discussion}

Deep learning with 3D data has not been researched nearly as much as with images. Therefore, it is necessary to select several frameworks for 3D object recognition which promises the best results for investigated objects in the factory based on above mentioned use cases.

Like most other object recognition architectures, VoxNet is not inherently rotationinvariant. For that reason, a data augmentation technique need to be implemented. During training, each model is rotated 12 times and trained on all copies. At test time, 
the output of the final fully-connected layer is pooled across several rotations of the input. In this way, VoxNet learns rotation invariance by sharing the same learned convolutional kernel weights across different rotations of the input voxel grid [32][33].

VoxNet is a huge progress in true $3 \mathrm{D}$ learning, but voxel grids still have a number of drawbacks. They lose resolution compared to point clouds, as several distinct points representing intricate structures will be binned into one voxel if they're close together. Voxel grids can yield unnecessarily high memory usage compared to point clouds in sparse environments, since they actively use memory to represent free and unknown space whereas point clouds contain only known points [33]. Finally, if an object is recognized, it must be put in the right position and proper orientation in the space. An automated procedure is necessary to support this matching of position and orientation.

\section{Conclusions and outlook}

The intention of this paper to close the gap between the real and virtual factory in the modern manufacturing industry by generation a Digital Twin of a built environment is presented. In particular, an industrial approach for a commercial service being in the implementation within www.OpenDESC.com to generate the Digital Twin based on fast scanning on a factory and subsequent object recognition is described. With the procedure described, which covers all required use cases, it is possible that a Digital Twin is created in few days and with little effort. Likewise, an update of existing models is feasible. The companies do not necessarily need their own experts with programming skills, but are supplied with a complete simulation model through the comprehensive service concept. Correspondingly, planning can be carried out more efficiently and flexibly, and productivity and quality increases are created.

\section{Acknowledgement}

The research project "DigiTwin - Efficient Generation of a Digital Twin in the Manufactoring" is supported by the German Federal Ministry of Education and Research (BMBF) within the Framework Concept "KMU innovativ". Authors are responsible for the contents of this publication.

\section{References}

[1] N. N., VDI Richtlinie 4499, Digitale Fabrik, Blatt 1: Grundlagen und Planung, Beuth-Verlag, Berlin, 2008.

[2] S. Wenzel et al., Qualitätskriterien für die Simulation in Produktion und Logistik. Springer, Berlin, 2008.

[3] S. Spieckermann, Diskrete, ereignisorientierte Simulation in Produktion und Logistik - Herausforderungen und Trends, Simulation und Visualisierung 2005 - SimVis. SCS Publishing House, Erlangen, 2005, pp. 314.

[4] M. Rabe, S. Spieckermann, S. Wenzel, Verifikation und Validierung für die Simulation in Produktion und Logistik: Vorgehensmodelle und Techniken, Springer-Verlag, Berlin, 2008.

[5] P. Nyhuis, H.-P. Wiendahl, Logistische Kennlinien: Grundlagen, Werkzeuge und Anwendungen, SpringerVerlag, Berlin, 2012.

[6] S. Bierschenk, A. Ritter, T. Kuhlmann, Stand der Digitalen Fabrik bei kleinen und mittelständischen Unternehmen. Studie, Fraunhoder, Stuttgart, 2005.

[7] U. Weber, N.A. Hong, V. Schäfer, P. Peters, S. Vettermann, S., Synced Factory Twins: Next Lever Digital Manufacturing, ProduktDataJournal, Issue 2, 2015, pp. 8-13. 
[8] S. Bondar, C. Ruppert and J. Stjepandić, Ensuring data quality beyond change management in virtual enterprise, International Journal of Agile Systems and Management, Vol. 7, 2014, Nos. 3/4, pp. 304-323.

[9] Z. Ma, S. Liu, A review of 3D reconstruction techniques in civil engineering and their applications, Advanced Engineering Informatics, Vol. 37, 2018, pp. 163-174.

[10] Z. Pučko, N. Šuman, D. Rebolj, Automated continuous construction progress monitoring using multiple workplace real time 3D scans, Advanced Engineering Informatics, Vol. 38, 2018, pp. 28-40.

[11] K. Kawashima, S. Kanai and H. Date, As-built modeling of piping system from terrestrial laser-scanned point clouds using normal-based region growing, Journal of Computational Design and Engineering, Vol. 1, 2013, pp. 13-26.

[12] F. Elgh, Automated Engineer-to-Order Systems A Task Oriented Approach to Enable Traceability of Design Rationale, Int. J. Agile Systems and Management, vol. 7, Nos 3/4, 2014, pp 324-347.

[13] J. Stjepandić, E. Ostrosi, A.-J. Fougères, M. Kurth, Modularity and Supporting Tools and Methods, in: J. Stjepandić et al. (eds.): Concurrent Engineering in the $21^{\text {st }}$ Century: Foundations, Developments and Challenges, Springer International Publishing Cham, 2015, pp. 389-420.

[14] V. Pătrăucean, I. Armeni, M. Nahangi, J. Yeung, I. Brilakis and C. Haas, State of research in automatic as-built modelling, Advanced Engineering Informatics, Vol. 29 (2) , 2015, pp. 162-171.

[15] A. Biahmou, C. Emmer, A. Pfouga and J. Stjepandić, Digital master as an enabler for industry 4.0, Advances in Transdisciplinary Engineering, Vol. 4, 2016, pp. 672-681.

[16] E. Rauch, P. Dallasega and D.T. Matt, Distributed manufacturing network models of smart and agile mini-factories, Int. Journal of Agile Systems and Management, Vol. 10, 2017, Nos. 3/4, pp. 185-205.

[17] C. Emmer, A. Fröhlich and J. Stjepandić, Advanced engineering visualization with standardized 3D formats, IFIP Advances in Information and Communication Technology, Vol. 409, Springer, Berlin Heidelberg, 2013, pp. 584-595.

[18] J. Bischoff, C. Taphorn, D. Wolter, N. Braun, M. Fellbaum, Goloverov, A.; Ludwig, S.; Hegmanns, Tobias; Prasse, Christian; Michael Henke, Michael ten Hompel, Döbbeler, Frederik; Fuss, Emanuel; Kirsch, Christopher; Mättig, Ben; Braun, S.; Guth, M.; Kaspers, M.; D. Scheffler, Erschließung der Potenziale der Anwendungen von „Industrie 4.0“ im Mittelstand. Fraunhofer, Dortmund, 2015, http://publica.fraunhofer.de/documents/N-356542.html, accessed Jan 202019.

[19] S. Jensen, Eine Methodik zur teilautomatisierten Generierung von Simulationsmodellen aus Produktionsdatensystemen am Beispiel einer Job Shop Fertigung. Kassel University Press, Kassel, 2007.

[20] B. Denkena, F. Winter, S. Hauck, Anlernen von ablauforientierten Simulationsmodellen. ProductivITy 21(3): , 2016, pp. 52-54.

[21] G. Schuh, R. Anderl, J. Gausemeier, M. Hompel, W. Wahlster, Industrie 4.0 Maturity Index - Die digitale Transformation von Unternehmen gestalten. Herbert Utz, München, 2017.

[22] S. Bergmann, Automatische Generierung adaptiver Modelle zur Simulation von Produktionssystemen, Diss., Technische Universität Ilmenau, 2013.

[23] P. Lorenz, T. Schulze, Layout based model generation. Proceedings of the 1995 Winter Simulation Conference. Arlington, VA, USA, 1995, pp. 728-735.

[24] R. Splanemann, Teilautomatisierte Generierung von Simulationsmodellen aus systemneutrale definierten Unternehmensdaten, Diss., Universität Bremen, 1995.

[25] C.C. Wuttke, Mehrfachnutzung von Simulationsmodellen in der Produktionslogistik. Schriftreihe Produktionstechnik - Band 20. Universität des Saarlandes, Saarbrücken, 2000.

[26] C. Selke, Entwicklung von Methoden zur automatischen Simulationsmodellgenerierung, Diss., Technische Universität München, 2005.

[27] C. Zenner, Durchgängiges Variantenmanagement in der Technischen Produktionsplanung. Schriftreihe Produktionstechnik - Band 37. Universität des Saarlandes, Saarbrücken, 2006.

[28] G. Šagi, Z. Lulić and I. Mahalec, Reverse Engineering, in: J. Stjepandić et al. (eds.) Concurrent engineering in the 21st century: Foundations, developments and challenges, Springer International Switzerland, 2015, pp. 319-353.

[29] Le Zhang, Jian Sun, and Qiang Zheng, 3D Point Cloud Recognition Based on a Multi-View Convolutional Neural Network, Sensors, 2018, 18, 3681; doi:10.3390/s18113681.

[30] J. Dekhtiar, A. Durupt, M. Bricogne, B. Eynard, H. Rowson, D. Kiritsis, Deep learning for big data applications in CAD and PLM - Research review, opportunities and case study, Computers in Industry, Vol. 100, Sep 2018, pp. 227-243.

[31] C. Wang, M. Cheng, F. Sohel , M. Bennamoun, J. Li, NormalNet: A voxel-based CNN for 3D object classification and retrieval, Neurocomputing, Vol. 323, 2019, pp. 139-147.

[32] D. Maturana and S. Scherer, VoxNet: A 3D Convolutional Neural Network for Real-Time Object Recognition, IEEE International Conference on Intelligent Robots and Systems, 2015December,7353481, pp. 922-928.

[33] M. Garimella and P. Naidu, Beyond the pixel plane: sensing and learning in 3D, The Gradient, 24. Aug 2018, https://thegradient.pub/beyond-the-pixel-plane-sensing-and-learning-in-3d/. 